\title{
Demonstration of a High-Throughput Tensile Testing Technique Using Femtosecond Laser-Fabricated Tensile Bars in AISI 316 and Additively Manufactured Grade 91 Steel
}

\author{
CAYLA HARVEY (i), ${ }^{1,5}$ AVERY J. TORREZ, ${ }^{2}$ SEBASTIAN LAM, ${ }^{3}$ \\ HYOSIM KIM, ${ }^{4}$ STUART A. MALOY ${ }^{4}$ and JONATHAN G. GIGAX ${ }^{2,6}$ \\ 1.-Chemical and Materials Engineering, University of Nevada, Reno, NV, USA. 2.-Center for \\ Integrated Nanotechnologies, Los Alamos National Laboratory, Los Alamos, NM, USA. \\ 3.-Department of Nuclear Engineering, University of California, Berkeley, CA, USA. 4.-Materials \\ Science and Technology Division, Los Alamos National Laboratory, Los Alamos, NM, USA. \\ 5.—e-mail: caylaharvey@nevada.unr.edu. 6._e-mail: jgigax@lanl.gov
}

\begin{abstract}
Femtosecond laser machining offers a fast, low damage route to fabricating features on the order of a few micrometers to millimeters. Within this broad application space, mechanical testing samples on this scale provides a combination of region selectivity and a bulk-like response. Use of such mechanical test specimens, however, is relatively unexplored compared to microscale and macroscale testing. The present study showcases the benefit of combining efficient specimen fabrication and testing on the mesoscale for extracting a more comprehensive picture of material mechanical properties. Moreover, a material with a complex and heterogeneous microstructure, additively manufactured grade 91 , has also been studied to highlight the considerations needed and limitations of testing on this scale.
\end{abstract}

\section{INTRODUCTION}

Femtosecond laser cutting presents a novel solution for machining structures intended for mechanical testing on the mesoscale. Preliminary studies show that the damage regions created by the method are minimal and ablation rates are 4-6 orders of magnitude higher than that of a traditional Ga focused ion beam (FIB) making it a prime candidate for the time-efficient machining of mesoscale mechanical test specimens. ${ }^{1-5}$ Methods like FIB can machine out mechanical test specimens on the order of microns, while wire EDM and waterjet can operate on the millimeter length scale. ${ }^{6}$ Although plasma FIB is a more common technique, removal rates at larger feature sizes (i.e., 100s of $\mu \mathrm{m}$ ) are limited. Mesoscale testing can be a viable tool to bridging the length scale gaps for materials characterization and, in some cases, the optimal technique. Small-scale mechanical test specimens produced in the FIB fall short of producing bulk material responses when samples are coarser

(Received July 20, 2021; accepted October 1, 2021; published online October 26, 2021) grained due to the inability to sample multiple grains during a single test. Not only do femtosecond laser techniques excel at the mesoscale, but they also have rapid turnaround times for sample production due to the high ablation rates.

Conventional laser machining, using continuous wave or long pulse duration lasers, has not been utilized for the creation of small-scale mechanical test specimens because of the large damage regions created by the beam. Momma et al. suggests that the high fluence of longer pulsed lasers $\left(t_{\text {pulse }}>\right.$ $10 \mathrm{ps}$ ) caused the beam energy to first melt the material until the vaporization temperature was reached..$^{5}$ The time scale of these lasers is long enough for a thermal wave to melt significant portions of the material, causing a large heat affected zone (HAZ). With femtosecond lasers, which operate in a low fluence regime, free electrons primarily absorb the beam energy. Since energy conduction between the electrons and lattice is low, the vaporization temperature can be easily reached without significant damage to the lattice. A further study by Le Harzic et al. correlates well with the effects of the proposed mechanism finding that, for 
the same pulse energy, a nanosecond laser $\left(t_{\text {pulse }}=\right.$ $7-8 \mathrm{~ns})$ and a femtosecond laser $\left(t_{\text {pulse }}=150 \mathrm{fs}\right)$ exhibited a HAZ of $40 \mu \mathrm{m}$ and $2 \mu \mathrm{m}$, respectively. ${ }^{2}$ Similar studies also found damage regions for femtosecond lasers amounting to $5 \mu \mathrm{m}$ parallel to the beam path. ${ }^{2-5}$

Despite the HAZ being present in mechanical test specimens in small volumes after femtosecond laser processing, this effect does not seem to change mechanical data when accounted for. Gigax et al. used femtosecond machining to create tensile samples for a coarse-grained metal which resulted in good correlation with that of bulk testing. 7 The HAZ in this study would have accounted for an insignificant fraction of the total gauge volume, meaning that its effects could be ignored. Another study also looked at the effects of the femtosecond laser process on the production of micropillars. ${ }^{8} \mathrm{~A}$ FIB was used to mill away $5 \mu \mathrm{m}$ of the suspected HAZ after the laser processing, which created results similar to a purely FIB-based approach during compression testing. Since the mechanical test specimens were on the length scale of the HAZ, its effects could not be ignored and needed to be removed.

The principal focus of this study is to showcase a rapid mechanical evaluation technique using femtosecond laser machining. Here, $316 \mathrm{~L}$ and additively manufactured grade 91 steels were selected and mesoscale tensile specimens fabricated and tested. At the outset, the goal was to not only demonstrate a rapid evaluation process but also a testing paradigm that couples both region selectivity and a bulk-like response. While the $316 \mathrm{~L}$ foil was chosen as a benchmark for the process, the additively manufactured grade 91 (AM91) was a good candidate as it contains a very heterogeneous microstructure.

Grade 91 steel, which belongs to a group of creep strength-enhanced ferritic steels, is a material that exhibits superior radiation resistance. ${ }^{9-12}$ However, it lacks strength at temperatures above $400^{\circ} \mathrm{C}^{12-17}$ AM91 has shown promising mechanical properties at temperatures between $300^{\circ} \mathrm{C}$ and $600^{\circ} \mathrm{C}$ for bulk mechanical tensile tests. ${ }^{13,14}$ The complex microstructure containing fine- and coarse-grained regions, in addition to a mixture of ferrite, bainite, and martensite, give rise to these superior properties at high temperatures.

While it is expected that large prints, containing a representative portion of this microstructure, should exhibit this level or performance regardless of structure or form, this may not necessarily apply to smaller structures. As an example, consider a lattice print containing struts less than $0.2 \mathrm{~mm}$ in thickness or tubing with comparable wall thicknesses. For these structures, the volume may undersample the microstructure reported in the as-deposited builds. Some portions of the print may contain a high fraction of martensite or small grains, leading to lower than expected ductility. Other regions may contain only large grains and exhibit low strength.

In this study, we leverage the high throughput nature of femtosecond laser machining and an inhouse-developed mechanical stage to explore the mechanical properties of a heterogeneous material, AM91, on the mesoscale. By pulling several samples along one direction of the print, the large dataset provides a bounding limit of the mechanical response on the selected length scale. 316L tensile specimens were tested first, to benchmark the selected process and to ensure repeatability and accuracy.

\section{METHODS AND MATERIALS}

AISI 316 foil samples were purchased from Goodfellow and have a nominal composition as listed in Table I. Additively manufactured grade 91 prints with a composition given in Table I were fabricated using the equipment and process parameters outlined in. ${ }^{13}$

AM91 specimens for tensile fabrication were sectioned from the print along the build direction shown in Fig. 1, and polished to a final solution of $0.25 \mu \mathrm{m}$ diamond on both sides of the sectioned piece to a nominal thickness of $0.15 \mathrm{~mm}$. The $316 \mathrm{~L}$ foil samples were cut in the as-received condition. All the samples for electron backscatter diffraction (EBSD) were polished to a final solution of $0.04-\mu \mathrm{m}$ silica. EBSD with automated phase indexing was performed in a FEI Inspect ${ }^{\mathrm{TM}}$ dual beam scanning electron microscope (SEM) with an EDAX TSL Hikari camera. The EBSD measurements were carried out at $20 \mathrm{kV}$, a current of $1.6 \mathrm{nA}$, and step size of $0.15 \mu \mathrm{m}-0.20 \mu \mathrm{m}$.

Tensile specimens were machined using the FemtoScribe system at the Center for Integrated Nanotechnologies. ${ }^{6}$ All the tensile specimens were cut using a laser wavelength of $1035 \mathrm{~nm}$, pulse width of $300 \mathrm{fs}$, pulse energy of $16 \mu \mathrm{J}$, and a repetition rate of $50 \mathrm{kHz}$. A $\times 5$ near-IR objective was used to focus the beam to $\mathrm{a} \sim 20 \mu \mathrm{m}$ spot size. A nominal gauge

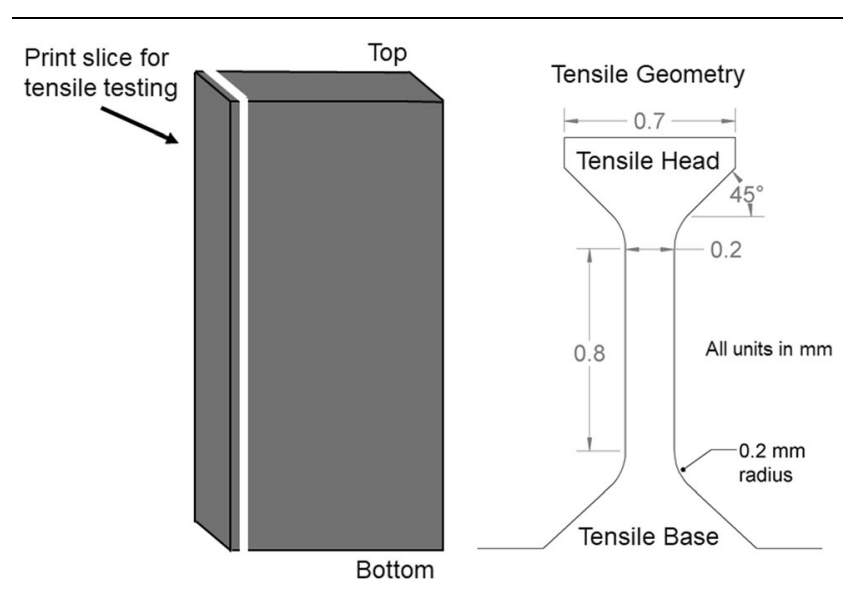

Fig 1. The AM91 print used for tensile testing. Schematic of the tensile bar geometry used for testing in this study shown to the right. 


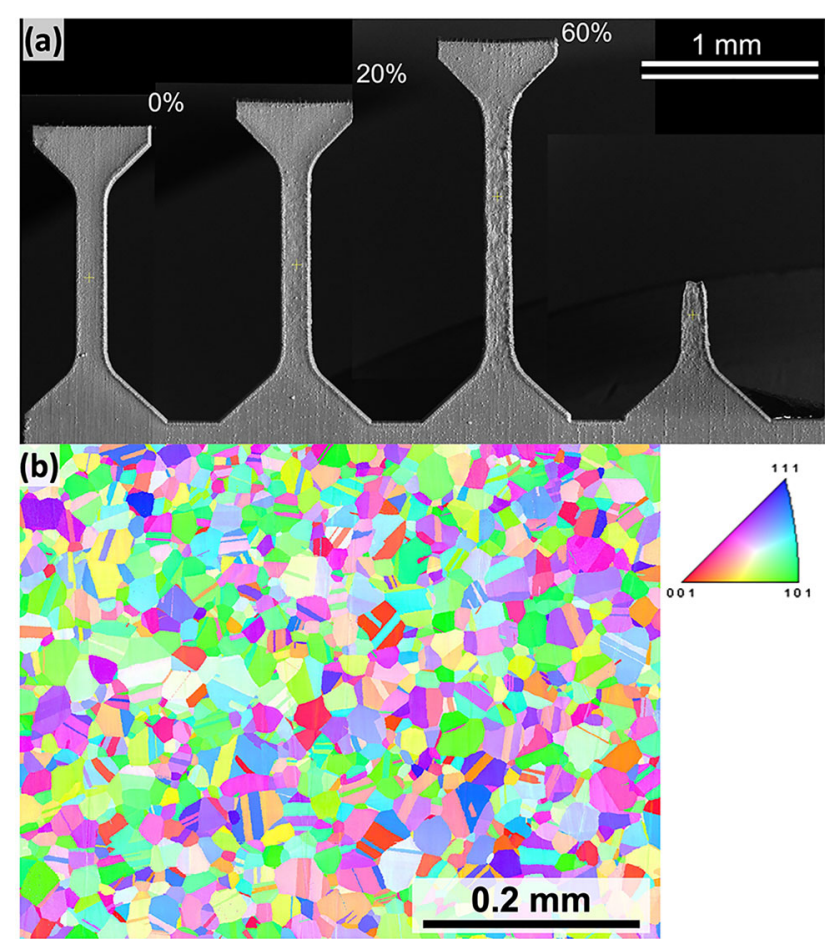

Fig 2. (a) Femtosecond lasermachined $316 \mathrm{~L}$ tensile bars pulled to varying strain levels. (b) EBSD IPF map of the 316L sample.

area of $0.2 \mathrm{~mm}^{2}$ for the $316 \mathrm{~L}$ tensile bars and 0.15 $\mathrm{mm}^{2}$ for the AM91 tensile bars, and a gauge length of $0.8 \mathrm{~mm}$ was selected. The taper of the samples was measured to be approximately $5^{\circ}$. A baseline comparison to a larger-scale $316 \mathrm{~L}$ specimen was performed to verify the mechanical response of the 316L mesotensile bars. The larger 316L specimens were fabricated to nominal gauge area of $2.4 \mathrm{~mm}^{2}$ and a gauge length of $13 \mathrm{~mm}$. All the specimens were pulled at an initial strain rate of $10^{-3} \mathrm{~s}^{-1}$ and at a constant displacement rate throughout the test. For mesoscale mechanical testing, an in-housedeveloped system was used to pull the tensile specimens under an optical microscope. Compliance of the mechanical load frame was measured by testing a material with known properties, without inducing yielding, and applying this correction when calculating the strain.

\section{RESULTS}

\section{L Grade Steel}

While the use of femtosecond laser machining has been explored in a variety of material systems for a range of applications, mechanical testing has received less attention. Before testing a complicated material, such as AM91, it is important to benchmark the preparation technique and in-house-built systems. AISI 316L stainless steel was selected as the material of choice for benchmarking due to its ubiquity in commercial applications and large material database.
Tensile bars fabricated in the $316 \mathrm{~L}$ foil are shown in Fig 2a. The left tensile bar is a representative specimen for the geometry selected for the 316L foil tests. For this sample, the gauge area is fixed to 0.2 $\mathrm{mm} \times 0.1 \mathrm{~mm}\left(0.2 \mathrm{~mm}^{2}\right)$ with a gauge length of 0.7 $\mathrm{mm}$. Shoulder cut-outs were included in the design to prevent stress concentrations near the base or top of the tensile sample. Unlike macroscale testing where it is possible to seat a tensile bar in the grippers and ensure good alignment prior to pulling, the approach to mesoscale mechanical testing taken here does not allow for this alignment. This is due primarily to the prohibitively small size of the tensile specimen and the relative insensitivity of human operators to manipulate such a specimen and avoid significant deformation during handling. Instead, tensile bars are single-ended cuts with one free end of the tensile bar for gripping and a fixed end on the substrate of the sample. The selection of a $45^{\circ}$ angle provides a balance between a gripper contact area and a more accommodating geometry for small misalignments that are invariably present in spite of best efforts to avoid this.

Tensile bars pulled to $20 \%$ and $60 \%$ strain are shown to the right of the undeformed specimen in Fig. 2a. While there is some minor deformation in the tensile bar head at high strain levels, the majority of deformation is contained within the gauge volume bounded by the rounded shoulder cutouts and shown by the change in surface roughness. Representative fracture of the tensile bars, shown in the right-most specimen, clearly show ductile fracture contained in the designated tensile volume.

One advantage of mesoscale testing is the combination of two unique properties of the neighboring length scales: a bulk-like response with region selectivity. While the latter is easily accomplished with the femtosecond laser cutting technique used in this study, obtaining a bulk-like response requires some knowledge of the microstructure. Figure $2 b$ provides an EBSD inverse pole figure (IPF) map of the $316 \mathrm{~L}$ foil. Equiaxed grains from 10 to $40 \mu \mathrm{m}$ in diameter are present in the foil. There is also no strong texture in the microstructure, which could vary the tensile bar response dependent on the orientation of the gauge volume with respect to host material. For the gauge area selected, at least 15 grains are included, assuming tensile bars are fabricated with the largest grains measured. In the gauge volume, a minimum of 300 grains are loaded during a mesoscale tensile test, providing confidence that the response of the tensile bars will be bulk-like.

One of the unique advantages of the technique presented in this study is the rapid fabrication and testing of specimens. Each tensile bar required no more than 5 min of time from the start of femtosecond laser machining to the end of the tensile pull. The rapid nature of this test lends to an improved collection of data that is often needed when studying fracture behavior, especially in brittle materials. 


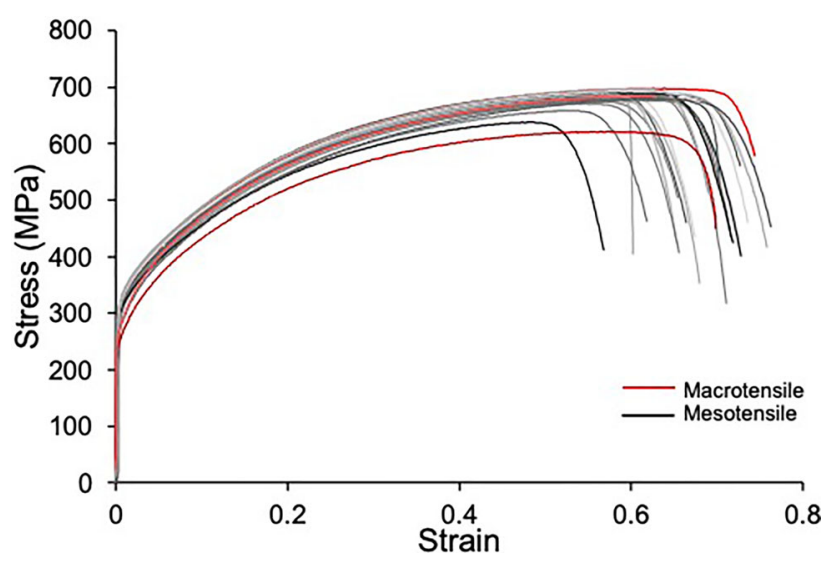

Fig 3. Collection of 19 engineering stress-strain curves of the $316 \mathrm{~L}$ tensile specimens pulled to fracture. Tensile bars not pulled to failure are not shown here.

Figure 3 showcases 19 engineering stress-strain curves obtained from $316 \mathrm{~L}$ tensile specimens pulled to fracture. Tensile specimens pulled partially to failure are not shown.

There are a few notable observations from the tests. First, most, if not all, curves align nicely, suggesting that the strain hardening behavior of the $316 \mathrm{~L}$ foil is independent of location in the foil itself and is indicative of a uniform microstructure. Moreover, the yield strength (305 $\mathrm{MPa} \pm 8.99$ $\mathrm{MPa})$ and ultimate tensile strength $(682 \mathrm{MPa} \pm$ 14.0 $\mathrm{MPa}$ ) exhibit a low standard deviation. The uniform $(57.8 \% \pm 5.58 \%)$ and total elongations $(68.8 \% \pm 4.89 \%)$ show slightly higher deviations. It is worth noting that two outlying stress-strain curves on the plot show a lower strain to failure than the average.

The majority of the curves fall within one standard deviation of the average value for the 316L foil. It is worth noting, however, that there are a few curves that fall outside this range. While it is expected that a few test specimens will be representative of the average, the results indicate the need to broaden the test matrix to include a sufficient number of tests to identify outliers.

\section{AM91}

AM91 was selected in this study due to its complex microstructure. A previous study on this print showed a microstructure containing fine- and coarse-grained regions, in addition to a mix of ferrite and bainite. The motivation for the present study is to explore the influence that this inhomogeneity might have on tensile bars at this scale. At the outset of our testing, it is important to note that the load frame used for pulling on these specimens had a maximum load limit of $20 \mathrm{~N}$. This corresponds to a maximum gauge area of $\sim 0.2 \mathrm{~mm}^{2}$. In anticipation of a wide variation in the tensile response, the gauge area for all specimens was limited to 0.15 $\mathrm{mm}^{2}$ to avoid exceeding the maximum load rating of the load frame.

Tensile bars were fabricated along the print direction to sample the homogeneity of the print properties from the bottom to the top of the print. An example of tensile bars fabricated in the AM91 is shown in Fig. 4a. The microstructure is shown in Fig. 4(b-d), with EBSD IPF maps showing large grains on the order of a few $100 \mu \mathrm{m}$, and fine grains around a few microns in diameter. Previous work examined the microstructure in greater detail noting the areas of relatively harder martensite and uniform distributions of precipitates present in the print. ${ }^{13,14}$ For the specimen geometry selected, only a few grain boundaries from coarse-grained regions may be contained within a tensile bar. The gauge area will consist of a mixture of fine and coarse grains or just one of these grain sizes depending on the location. This is unlike a macroscale tensile dogbone that will contain a mixture of fine- and coarse-grained regions in the gauge area.

This more varied microstructure within the tensile bars lead to a greater variation of the stressstrain response, as shown in Fig. 5. The yield strength average is $755 \mathrm{MPa} \pm 109 \mathrm{MPa}$, with an average ultimate tensile strength of $940 \mathrm{MPa} \pm 98.3$ $\mathrm{MPa}$. The results as a function of distance from the bottom of the print are shown in Fig. 5(b-e). The difference between batch 1 and batch 2 of tests is notable. Batch 1 was fabricated within $0.5 \mathrm{~mm}$ of the edge of the print, while batch 2 was located closer to the center of the print. Batch 1 exhibits a higher average yield strength (831 $\mathrm{MPa}$ vs. 681 MPa) than batch 2 .

\section{DISCUSSION}

Micro-tensile testing on the 316L steel shows the repeatability of this fabrication and test method for a material with relatively small grains to the gauge length. The results exhibit a bulk-like response, indicating tensile testing on the mesoscale is a viable route to obtaining tensile properties with a small volume of material. Coupled with femtosecond laser machining, mesoscale tensile testing is a highthroughput technique for evaluating the mechanical properties of materials.

For more challenging materials, such as AM91 with a larger variation in the microstructure, testing on this length scale offers more challenges. The tensile response is sensitive to the region of the microstructure selected and, in turn, produces upper and lower limits for the response of the material. How does the testing in this material system compare with a macroscale tensile test? Eftink et al. reported an average yield and ultimate tensile strength of $773 \mathrm{MPa}$ and $895 \mathrm{MPa}$, respectively. The average response of all mesoscale tensile testing is in good agreement with these results. 

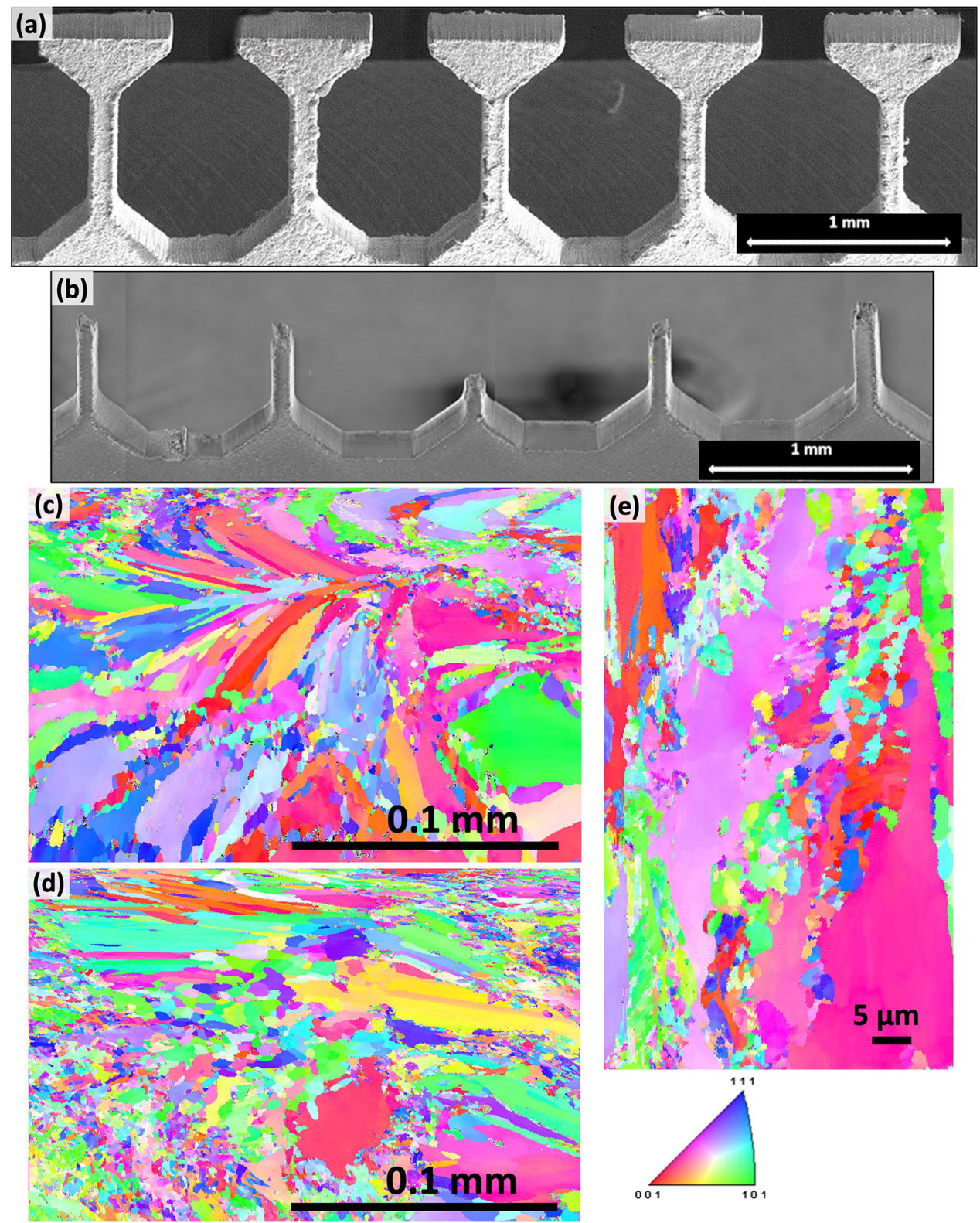

Fig 4. Femtosecond laser-fabricated AM91 tensile bars (a) before testing and (b) after deformation. (c, d) EBSD IPF maps of AM91, (c) and (d) show grain size and texture in two different areas on the bar, and (e) shows a higher magnification of fine grain features.

The largest disparity between the macro- and mesoscale tensile tests is in the elongation behavior. Here, very few tensile specimens achieve the uniform and total elongations of $10 \%$ and $20 \%$, respectively, observed in the macroscale tensile tests. The vast majority of specimens exhibit elongations of approximately half of these values. The biggest source of this difference lies in the sampled microstructure. A balance in strength and ductility in systems with both fine and coarse grains arises from the inclusion of both regions in the gauge area. ${ }^{18}$ If only fine grains are contained in the area, then it is expected that the response has a relatively high strength and low ductility compared to areas containing only coarse grains.
Additional considerations must also be made to contained phases. Martensite, a harder phase than ferrite or bainite, is expected to contribute to a higher strength in some samples. Ferritic grains, on the other hand, are expected to provide ductility with a lower strength. The results in this study indicate that a deliberate choice of mesotensile geometry must be made prior to testing in order to closely link the response on the mesoscale with that observed on the macroscale.

However, the results are not necessarily irrelevant to additive manufacturing. Many small-scale structures, such as support struts on lattice prints, are similar in dimension of the tensile specimens cut in this study. For these structures, it is 

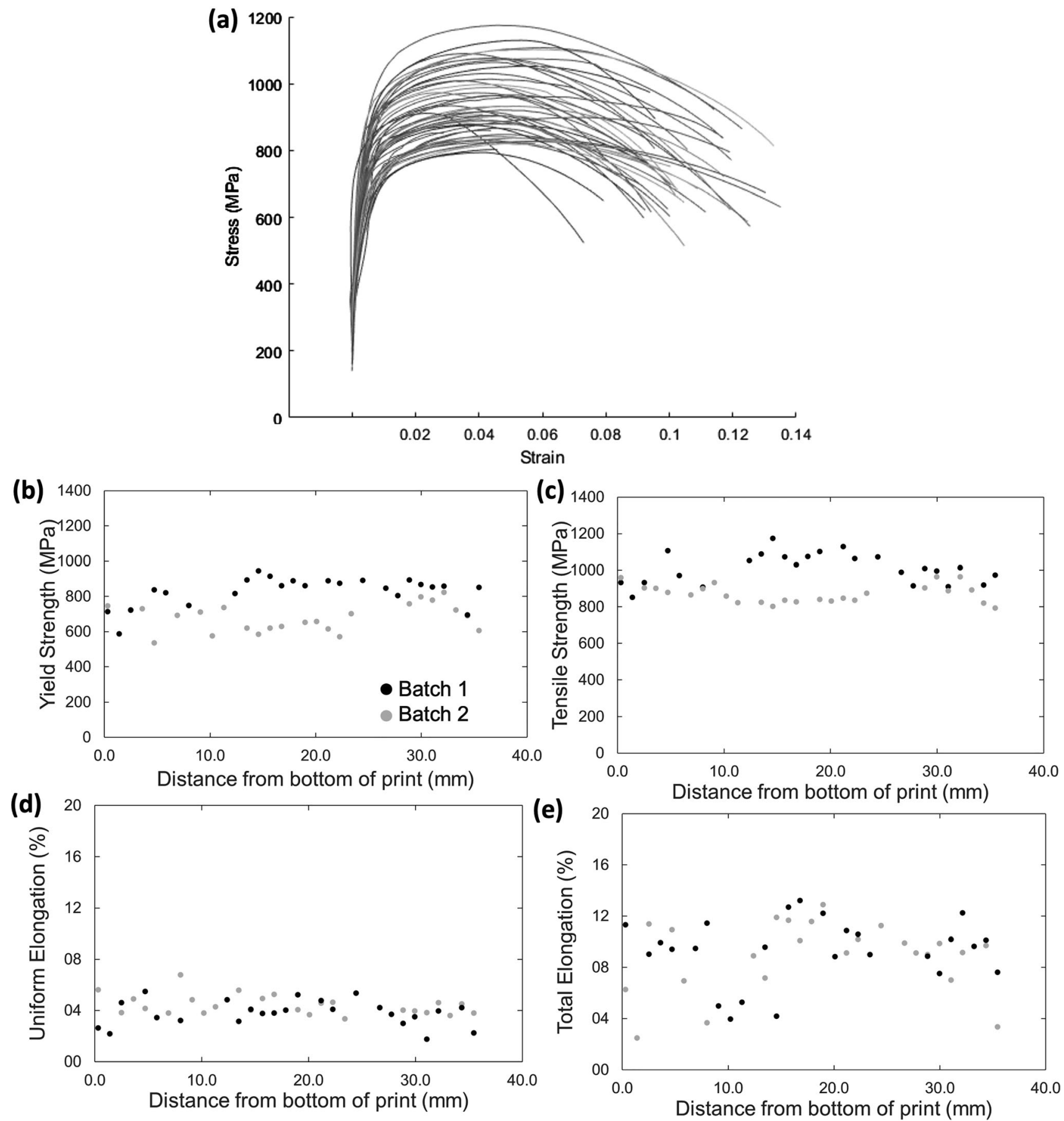

Fig 5. (a) Collection of 66 engineering stress-strain curves of the AM91 tensile specimens pulled to fracture, showing results for batch 1 and 2. Following graphs showing (b) yield strength, (c) tensile strength, (d) total elongation, and (e) uniform elongation as a function of distance from the bottom of the print.

important to consider the influence that an undersampled microstructure, compared to a larger print feature, might have on their mechanical properties. The testing here provides a picture of the bounds for the mechanical response expected for structures on this scale. Certain mesoscale tensile specimens exhibit a very low strength and comparatively poor ductility, likely due to a deleterious sampling of less ductile phases. On the other hand, some tensile specimens exhibit mechanical properties that reach or exceed those reported on the macroscale.

Additionally important to AM processing is the notable difference in strength between batch 1 and batch 2 . The location of batch 1 was within $1 \mathrm{~mm}$ of the edge of the print with the gauge volume spanning a distance $0.4-1.2 \mathrm{~mm}$ from the edge of the print. Batch 2, on the other hand, was located a 
Table I. Chemical composition of AISI 316L and additively manufactured grade 91 steels; all values are in wt.\%

\begin{tabular}{lcrrrrrrrrrrrrr}
\hline & $\mathbf{F e}$ & $\mathbf{C r}$ & $\mathbf{N i}$ & $\mathbf{M o}$ & $\mathbf{M n}$ & $\mathbf{S i}$ & $\mathbf{N b}$ & $\mathbf{V}$ & $\mathbf{C}$ & $\mathbf{N}$ & $\mathbf{S}$ & $\mathbf{P}$ \\
316L & Bal. & 16.76 & 10.14 & & 2.02 & 0.88 & 0.31 & - & - & 0.022 & 0.048 & 0.001 & 0.031 \\
AM91 & Bal. & 9.01 & 0.071 & 0.92 & 0.45 & 0.042 & 0.096 & 0.17 & 0.077 & 0.03 & 0.005 & 0.004 \\
\hline
\end{tabular}

larger distance away, approximately $3 \mathrm{~mm}$ from the edge of the print. Due to the rastering behavior of the laser beam, a larger amount of energy is expected to be deposited near the edge of a print as the direction is changed and the laser dwells in this region for a longer period of time. This would, in turn, lead to greater annealing of the microstructure and potentially improved qualities. It is important to note, however, that the average ductility of batch 1 and batch 2 are nearly identical, suggesting that there may be another influence not identified here warranting additional investigation.

There are some outstanding concerns that should be addressed with the technique. Although the viability of the technique was established with 316L foil, the damage from femtosecond laser cutting and impact of debris was not previously addressed. We do not explore the extent of damage caused by the technique, but rely on prior studies that have shown that the damage for metals is limited to a few micrometers below the surface. ${ }^{7,8,19}$ It is not expected that, for the laser parameters used here, the damage should significantly exceed prior findings. For the 316L foil and AM91, a laseraffected region a few micrometers below the surface would comprise less than $5 \%$ of the response, well within the variation observed from measurements. Due to the lower thermal conductivity of these metals, it is likely that the laser-affected regions are smaller and influence the response less than the conservative estimate.

Debris from femtosecond laser machining in air environments is unavoidable and often in the form of oxides of the host material. Some approaches to removing debris have been explored by the authors, such as sonication in water or ethanol, but were not used in the present study due to the reduction in throughput and additional risk of damaging specimens during this process. Is it necessary to remove debris from tensile specimens before testing? The oxide debris on the surface of the sample is not sintered and is loosely bound. Furthermore, pulling on the tensile specimen does not load the debris in such a way as to influence the deformation behavior, as would normally be expected during a compression test. It is not expected that the debris on the specimens should influence, to any significant degree, the tensile behavior of either sample studied. However, further testing is needed to quantify the extent should concerns continued to be raised.

\section{CONCLUSION}

Femtosecond laser machining and mesoscale tensile testing was demonstrated on two materials, $316 \mathrm{~L}$ and additively manufactured grade 91 . The $316 \mathrm{~L}$ foil was used as a baseline to benchmark the accuracy and reliability of the results obtained from mesoscale tensile specimens fabricated by femtosecond laser machining and tested on an in-housedesigned system. The strength and elongation properties of the tensile specimens did not vary more than $10 \%$ across more than 20 tensile pulls, owing to a homogeneous microstructure. A few notable outlying mechanical responses were identified within the test set and highlight the need for large test matrices to avoid an undersampled response.

Tensile testing on this scale was also applied towards additively manufactured grade 91 . Unlike the $316 \mathrm{~L}$ foil, AM91 had a very complicated microstructure with fine- and coarse-grained region and a mixture of ferrite, bainite, and martensite. Due to the test platform loading limitations, specimens with a gauge area of only $0.15 \mathrm{~mm}^{2}$ were machined. At this scale, a representative microstructure is not always included in the gauge volume as is expected from a macroscale test. While the strength of the specimens is in good agreement with macroscale tests, the elongation properties are found to be significantly less. The results show a significant amount of variation in the tensile response, arising from what grain sizes and phases are included.

The results show that some knowledge of the microstructure must be known prior to machining tensile specimens on the mesoscale. Moreover, a careful choice of tensile geometry must be made, as results are often compared to macroscale testing. However, not all tensile geometries must be made to include a representative sampling of the microstructure. Small additively manufactured structures that are on the same length scale as the tensile specimens in this study encounter these potential issues. The mesoscale tensile testing of AM91 provides bounds on the expected tensile response for structures on this scale.

\section{ACKNOWLEDGEMENTS}

This work was performed, in part, at the Center for Integrated Nanotechnologies, an Office of Science User Facility operated for the U.S. Department of Energy (DOE) Office of Science. Work was per- 
formed at Los Alamos National Laboratory. Los Alamos National Laboratory is an affirmative action/equal opportunity employer, and is operated by Triad National Security, LLC for the National Nuclear Security Administration of U.S. Department of Energy under contract 89233218CNA000001. CH acknowledges support from the DOE Integrated University Program Graduate Fellowship for this work.

\section{CONFLICT OF INTEREST}

The authors declare that they have no conflict of interest.

\section{REFERENCES}

1. M.P. Echlin, M. Straw, S. Randolph, J. Filevich, and T.M. Pollock, Mater. Charact. 100, 1. (2015).

2. R. Le Harzic, N. Huot, E. Audouard, C. Jonin, and P. Laporte, Appl. Phys. Lett. 80, 21. (2002).

3. P.B. Corkum, F. Brunel, N.K. Sherman, and T. SrinivasanRao, Phys. Rev. Lett. 61, 2886. (1988).

4. S. Valette, E. Audouard, R. Le Harzic, N. Huot, P. Laporte, and R. Fortunier, Appl. Surf. Sci. 239(3-4), 381. (2005).

5. C. Momma, B.N. Chichkov, S. Nolte, F. von Alvensleben, A. Tunnerman, H. Welling, and B. Wellegehausen, Optics Commun. 129(1-2), 134. (1996).
6. Q. McCulloch, J.G. Gigax, and P. Hosemann, JOM 72, 1694. (2020).

7. J.G. Gigax, A.J. Torrez, Q. McCulloch, H. Kim, S.A. Maloy, and N. Li, J. Mater. Res. 35, 2817. (2020).

8. J.G. Gigax, H. Vo, Q. McCulloch, M. Chancey, Y. Wang, S.A. Maloy, N. Li, and P. Hosemann, Scr. Mater. 170, 145. (2019).

9. J. Parker, Int. J. Pressure Vessels Piping 114-115, 76. (2014).

10. Z.Q. Fan, T. Hao, S.X. Zhao, G.N. Luo, C.S. Liu, and Q.F. Fang, J. Nucl. Mater. 434(1-3), 417. (2013).

11. R.L. Klueh, Int. Mater. Rev. 50, 287. (2005).

12. R.L. Klueh, and A.T. Nelson, J. Nucl. Mater. 371, 37. (2007).

13. B.P. Eftink, D.A. Vega, O. El-Atwani, D.J. Sprouster, J.S. Yoo, T.E. Steckley, E. Aydogan, C.M. Cady, M. Al-Sheikhly, T.J. Lienert, and S.A. Maloy, J. Nucl. Mater. 544 (2021). h ttps://doi.org/10.1016/j.jnucmat.2020.152723.

14. O. El-Atwani, B.P. Eftink, C.M. Cady, D.R. Coughlin, M.M. Schneider, and S.A. Maloy, Scr. Mater. 199, 113888. (2021).

15. R.L. Klueh, and J.M. Vitek, J. Nucl. Mater. 137(1), 44-50. (1985).

16. C. Xu, and M. Hackett, Terra Power HT9 Mechanical and Thermal Creep Properties (Springer, Cham, 2017).

17. C. Keller, Mater. Sci. Eng. A 527, 6758. (2010).

18. Y.M. Wang, and E. Ma, Acta Mater. 52, 1699. (2004).

19. M.P. Echlin, M.S. Titus, M. Straw, P. Gumbsch, and T.M. Pollock, Acta Mater. 124, 37. (2017).

Publisher's Note Springer Nature remains neutral with regard to jurisdictional claims in published maps and institutional affiliations. 